\title{
Quantum Fourier transform and tomographic Rényi entropic inequalities
}

\author{
M. A. Man'ko*, V. I. Man'ko ${ }^{\dagger}$
}

\begin{abstract}
Аннотация
Rényi entropy associated with spin tomograms of quantum states is shown to obey to new inequalities containing the dependence on quantum Fourier transform. The limiting inequality for the von Neumann entropy of spin quantum states and a new kind of entropy associated with quantum Fourier transform are obtained. Possible connections with subadditivity and strong subadditivity conditions for tomographic entropies and von Neumann entropies are discussed.
\end{abstract}

keywords: uncertainty relations, entropy, quantum tomography, quantum Fourier transform.

\section{Introduction}

In previous work [1], the probability-operator-symbol framework for quantum information was constructed. In this approach, quantum-information ingredients like qubits, qudits, and the operators providing the description and connection of the qudit states are given in the form of functions called the operator symbols. For the operator symbols, the product rule called the star-product is determined by using an integral nonlocal kernel [2]. For the both pure and mixed qudit states, the operator symbols of their density operators are standard probability-distribution functions. Since the qudit states for multipartite systems are described by standard probability distributions, all the characteristics of the distributions including Shannon entropy [3] and Rényi entropy [4] can be used to introduce in quantum information [1] the operator-symbol entropies like, e.g., operatorsymbol Rényi entropies, operator-symbol relative $q$-entropy. Since the Shannon entropy is the limiting case of the Rényi entropy, the corresponding analogs of the operator-symbol Shannon entropy and the properties of this entropy can be obtained in quantum information within the introduced operator-symbol framework.

In quantum mechanics and quantum information, there is a fundamental feature distinguishing their quantum nature from their classical counterparts, that is the uncertainty relations. The uncertainty relations by Heisenberg [5] and by Schrödinger [6] and Robertson [7,8] written for conjugate variables like positions and momenta were also accompanied by the so-called entropic

${ }^{*}$ P. N. Lebedev Physical Institute, Leninskii Prospect 53, Moscow 119991, Russia, e-mail: mmanko@sci.lebedev.ru

${ }^{\dagger}$ P. N. Lebedev Physical Institute, Leninskii Prospect 53, Moscow 119991, Russia, e-mail: manko@sci.lebedev.ru 
uncertainty relations. The entropic uncertainty relations for the continuous variables were written in the form of inequalities for Shannon entropy associated with the position and momentum probability densities in [9-11].

Within the operator symbol framework, in quantum information the subadditivity and strong subadditivity conditions were obtained for probability distribution describing qudit quantum states (called spin tomograms) [1]. Also some relations of the inequalities with subadditivity and strong subadditivity conditions for the von Neumann entropy were clarified. In fact, within the operator symbol framework, the tomographic map of unitary group $U(n)$ onto simplex was given and, in view of this map, the notion of Shannon entropy, Rényi entropy, and other entropies were introduced for unitary group.

The entropic uncertainty relations for finite-dimensional quantum systems were obtained in the form of inequalities for Shannon entropies associated with probability distributions related to measuring noncommuting observables in [12-16]. The uncertainty relations for Rényi entropy for the position and momentum distributions and for finite-dimensional systems with measuring observables related by quantum Fourier transform were obtained in [17]. There exist for bipartite and tripartite systems the known inequalities for the von Neumann entropy called subadditivity and strong subadditivity conditions $[18,19]$.

The aim of this work is to review the results of [1] and extend the study of entropic inequalities like subadditivity and strong subadditivity conditions obtained in the previous work on the probabilistic operator symbol framework in quantum information and to find new entropic inequalities for spin tomograms which are analogs of entropic inequalities discussed in [12-17]. For continuous variables, the entropic inequalities for quantum symplectic tomogram were discussed in [20-25]. The essential aspect of the new inequalities is that they are closely related to properties of quantum Fourier transform discussed in [26] and used in quantum information (see, for example, [27-29]). The quantum Fourier transform as an important ingredient plays a key role in quantum computing, quantum information processing. 1

We will get entropic inequalities which provide some constrains for unitary spin tomograms connecting them with $\ln N$, where $N$ is dimension of Hilbert space. The number $\ln N$ has appeared in entropic inequalities in earlier works $[12-14,16]$ but a new element of the present study is that $\ln N$ is directly associated with tomographic-probability distribution determining the qudit state in the probability operator symbol framework in quantum information.

\section{Spin tomograms}

Given an $N$-dimensional Hilbert space of spin system. One can interpret the Hilbert space either as the state space for one particle with spin $j=(N-1) / 2$ or in the case of product representation of number $N=n_{1} n_{2} \ldots n_{M}$ as the space of multipartite spin system with $j_{1}=\left(n_{1}-1\right) / 2, j_{2}=$ $\left(n_{2}-1\right) / 2, \ldots, j_{M}=\left(n_{M}-1\right) / 2$. The $N \times N$ density matrix $\rho$ of the quantum state can be represented by the unitary tomogram of the spin state [30].

In the case of spin state with $j=(N-1) / 2$, the tomogram is defined by the relation

$$
w(m, u)=\left\langle m\left|u^{\dagger} \rho u\right| m\right\rangle,
$$

${ }^{1}$ This work was initiated by illuminating discussions with Rui Vilela Mendes and we thank him for his help in obtaining the results presented here. 
where $\rho$ is the density matrix, $u$ is $N \times N$ unitary matrix and semi-integers $m=-j,-j+1, \ldots, j$ are values of spin projection on the $z$ axis. The tomogram $w(m, u)$ is nonnegative probability distribution function of random spin variable satisfying the normalization condition

$$
\sum_{m=-j}^{j} w(m, u)=1
$$

and the equality

$$
\int w(m, u) d u=1
$$

where $d u$ is Haar measure on unitary group with normalization

$$
\int d u=1 .
$$

The important property of tomogram (1) is that its connection with density matrix $\rho$ is bijective, i.e., $\rho \leftrightarrow w(m, u)$ [1]. This means that the quantum state is given if the tomogram is known [31,32].

\section{Quantum Fourier transform}

The symmetric unitary $N \times N$ matrix $F$ with matrix elements $F_{j k}=\frac{1}{\sqrt{N}} \exp \left(\frac{2 \pi i}{N} j k\right)(j, k=$ $0,1, \ldots, N-1)$, which are characters of irreducible representation of cyclic group $C_{N}$, can be used to provide invertible map of normalized complex vector $\vec{a}$ with components $a_{k}$ onto complex vectors $\vec{a}^{(f)}$ with components $a_{k}^{(f)}$ as follows:

$$
a_{k}^{(f)}=\sum_{j=0}^{N-1} F_{k j} a_{j}, \quad a_{k}=\sum_{j=0}^{N-1}\left(F^{\dagger}\right)_{k j} a_{j}^{(f)} .
$$

The matrix $F$ with matrix elements $F_{k j}$ satisfies the equality

$$
F^{N}=1 \text {. }
$$

The map (5) is called the quantum Fourier transform for cyclic group $C_{N}$.

If one uses the usual labels for spin projection $m=-j,-j+1, \ldots, j$, the operator $\hat{F}$ for quantum Fourier transform can be defined as

$$
\hat{F}|m\rangle=\sum_{m^{\prime}=-j}^{j} F_{m^{\prime} m}\left|m^{\prime}\right\rangle,
$$

where the symmetric matrix

$$
F_{m^{\prime} m}=\left\langle m^{\prime}|\hat{F}| m\right\rangle
$$

has the form

$$
F_{m^{\prime} m}=\frac{1}{\sqrt{N}}\left(\begin{array}{ccccc}
1 & 1 & 1 & \cdots & 1 \\
1 & a & a^{2} & \cdots & a^{N-1} \\
1 & a^{2} & a^{4} & \cdots & a^{N-2} \\
\cdots & \cdots & \cdots & \cdots & \cdots \\
1 & a^{N-1} & a^{N-2} & \cdots & a
\end{array}\right), \quad a=\exp \left(\frac{2 \pi i}{N}\right)
$$


Thus the unitary operator of quantum Fourier transform reads

$$
\hat{F}=\sum_{m=-j}^{j} \sum_{m^{\prime}=-j}^{j} F_{m^{\prime} m}\left|m^{\prime}\right\rangle\langle m| .
$$

In view of (6) , one has $(\hat{F})^{N}=\hat{1}$, where $\hat{1}$ is the identity operator.

\section{Shannon and Rényi tomographic entropies}

Following standard definitions of probability theory, one can introduce Shannon [3] tomographic entropy [1,33] and Rényi [4] tomographic entropy [1]. The Shannon tomographic entropy (operatorsymbol Shannon entropy) is the function on unitary group

$$
H_{u}=-\sum_{m=-j}^{j} w(m, u) \ln w(m, u) .
$$

The Rényi tomographic entropy (operator symbol Rényi entropy) is also the function on the unitary group and it depends on extra parameter

$$
R_{u}=\frac{1}{1-q} \ln \left(\sum_{m=-j}^{j}\left(w((m, u))^{q}\right)\right.
$$

Likewise for two spin tomograms $w_{1}(m, u)$ and $w_{2}(m, u)$, we define the operator symbol relative $q$-entropy

$$
H_{q}\left(w_{1}(u) \mid w_{2}(u)\right)=-\sum_{m=-j}^{j} w_{1}(m, u) \ln _{q} \frac{w_{2}(m, u)}{w_{1}(m, u)}
$$

with

$$
\ln _{q} x=\frac{x^{1-q}-1}{1-q}, \quad x>0, \quad q>0, \quad \ln _{q \rightarrow 1} x=\ln x .
$$

The relative tomographic $q$-entropy is nonnegative.

For $q \rightarrow 1, R_{u} \rightarrow H_{u}$ and the operator symbol relative $q$-entropy becomes operator symbol relative entropy

$$
H\left(w_{1}(u) \mid w_{2}(u)\right)=-\sum_{m=-j}^{j} w_{1}(m, u) \ln \frac{w_{2}(m, u)}{w_{1}(m, u)} .
$$

As was shown in [1], the minimum over unitary group of the operator symbol Rényi entropy is equal to quantum Rényi tomographic entropy

$$
\min R_{u}=\frac{1}{1-q} \ln \operatorname{Tr} \rho^{q} .
$$

Also the relative entropy (13) is nonnegative function for any admissible deformation parameter $q$.

The minimum of entropy $H_{u}$ given by (11) over the unitary group is equal to von Neumann entropy [1,33], i.e.,

$$
\min H_{u}=-\operatorname{Tr} \rho \ln \rho .
$$




\section{Shannon entropic inequalities in measuring noncommutative observables}

In this section, we review known entropic inequalities [12-16] which appear in the problem of measuring two observables $\hat{A}$ and $\hat{B}$ in finite Hilbert space.

Let the spectral decompositions of Hermitian operators $\hat{A}$ and $\hat{B}$ read

$$
\hat{A}=\sum_{k} A_{k}\left|a_{k}\right\rangle\left\langle a_{k}\left|, \quad \hat{B}=\sum_{k} B_{k}\right| b_{k}\right\rangle\left\langle b_{k}\right|, \quad k=1, \ldots, N,
$$

where $A_{k}$ and $B_{k}$ are eigenvalues of the observables and $\left|a_{k}\right\rangle$ and $\left|b_{k}\right\rangle$ are their orthonormal systems of eigenvectors.

For pure state $|\psi\rangle$, one has two probability distributions

$$
p_{k}=\left|\left\langle a_{k} \mid \psi\right\rangle\right|^{2}, \quad q_{k}=\left|\left\langle b_{k} \mid \psi\right\rangle\right|^{2} .
$$

The corresponding Shannon entropies connected with these two distributions read

$$
H_{p}=-\sum_{k} p_{k} \ln p_{k}
$$

and

$$
H_{q}=-\sum_{k} q_{k} \ln q_{k} .
$$

They satisfy the inequality found in [34]

$$
H_{p}+H_{q} \geq-2 \ln \frac{1}{2}(1+c),
$$

where the bound $c$ is determined by maximum values of scalar product modulus

$$
c=\max _{j, k}\left|\left\langle a_{j} \mid b_{k}\right\rangle\right| .
$$

In [35] the inequality was conjectured to be improved

$$
H_{p}+H_{q} \geq-2 \ln c
$$

and in [12] it was proved.

For the case of observables $\hat{A}$ and $\hat{B}$ with eigenvectors providing mutually unbised bases $\left|a_{k}\right\rangle, \mid$ $\left.b_{k}\right\rangle$ (see $\left.[14,26]\right)$, i.e.,

$$
\max \left|\left\langle a_{i} \mid b_{j}\right\rangle\right|=\frac{1}{\sqrt{N}}
$$

inequality (24) reads [14]

$$
H_{p}+H_{q} \geq \ln N
$$

Thus the dimensionality of Hilbert space $N$ appears in the entropic inequality.

The problem of mutually unbiased bases is related to geometry of finite Hilbert spaces [36,37]. It was widely discussed in connection with constructing the Wigner function for finite Hilbert space and quantum cryptography (see, for example, [27,38-41]). 
The entropic inequalities for Shannon entropy can be also obtained in studying the problem of measuring several noncommutative observables with orthonormal sets of eigenvectors which satisfy the condition (25) (see [14,15]). In [13] the entropic inequalities for Tsallis entropy related to continuous variables were obtained on the base of Sobolev inequalities while in [17] the analogous entropic uncertainty relations for Rényi entropy both for finite Hilbert space and for continuous variables were presented.

\section{Known inequalities for bipartite and tripartite systems}

The operator symbol entropies satisfy some known inequalities found in [1]. For example, if the spin system is bipartite, i.e., one has spin $j_{1}$ and $j_{2}$, the basis in tensor product space reads

$$
\left|m_{1} m_{2}\right\rangle=\left|m_{1}\right\rangle\left|m_{2}\right\rangle \text {. }
$$

In this case, the tomogram is the joint-probability distribution of two random spin projections $m_{1}=-j_{1},-j_{1}+1, \ldots, j_{1}$ and $m_{2}=-j_{2},-j_{2}+1, \ldots, j_{2}$ depending on $\left(2 j_{1}+1\right)\left(2 j_{2}+1\right) \times\left(2 j_{1}+\right.$ 1) $\left(2 j_{2}+1\right)$ unitary matrix $u$. The tomogram reads

$$
w\left(m_{1}, m_{2}, u\right)=\left\langle m_{1} m_{2}\left|u^{\dagger} \rho(1,2) u\right| m_{1} m_{2}\right\rangle
$$

where $\rho(1,2)$ is the density matrix of bipartite system with matrix elements

$$
\rho(1,2)_{m_{1} m_{2}, m_{1}^{\prime} m_{2}^{\prime}}=\left\langle m_{1} m_{2}|\rho(1,2)| m_{1}^{\prime} m_{2}^{\prime}\right\rangle .
$$

For the tomogram, one can introduce the Shannon entropy $H_{12}(u)$ as

$$
H_{12}(u)=-\sum_{m_{1}=-j_{1}}^{j_{1}} \sum_{m_{2}=-j_{2}}^{j_{2}} w\left(m_{1}, m_{2}, u\right) \ln w\left(m_{1}, m_{2}, u\right)
$$

and the entropy satisfies the subadditivity condition for all elements of the unitary group

$$
H_{12}(u) \leq H_{1}(u)+H_{2}(u)
$$

where $H_{1}(u)$ and $H_{2}(u)$ are Shannon entropies associated with subsystem tomograms

$$
w_{1}\left(m_{1}, u\right)=\sum_{m_{2}=-j_{2}}^{j_{2}} w\left(m_{1}, m_{2}, u\right)
$$

and

$$
w_{2}\left(m_{2}, u\right)=\sum_{m_{1}=-j_{1}}^{j_{1}} w\left(m_{1}, m_{2}, u\right)
$$

as follows:

$$
H_{k}(u)=-\sum_{m_{k}=-j_{k}}^{j_{k}} w_{k}\left(m_{k}, u\right) \ln w_{k}\left(m_{k}, u\right), \quad k=1,2
$$


From this inequality, in view of the relation between the von Neumann and operator symbol entropies, follows the known inequality [1], which is subadditivity condition for corresponding von Neumann entropy for bipartite system

$$
S_{12} \leq S_{1}+S_{2}
$$

where

$$
S_{k}=-\operatorname{Tr} \rho_{k} \ln \rho_{k}, \quad k=1,2
$$

and

$$
\rho_{1}=-\operatorname{Tr}_{2} \rho(1,2), \quad \rho_{2}=-\operatorname{Tr}_{1} \rho(1,2) .
$$

For tripartite spin system with spins $j_{1}, j_{2}, j_{3}$ and density matrix $\rho(1,2,3)$, the spin tomogram reads

$$
w\left(m_{1}, m_{2}, m_{3}, u\right)=\left\langle m_{1} m_{2} m_{3}\left|u^{\dagger} \rho(1,2,3) u\right| m_{1} m_{2} m_{3}\right\rangle .
$$

One associates with this tomogram the Shannon entropy $H_{123}(u)$. This entropy satisfies inequality, which is the strong subadditivity condition on the unitary group. It reads [1]

$$
H_{123}(u)+H_{2}(u) \leq H_{12}(u)+H_{23}(u),
$$

where

$$
H_{123}(u)=-\sum_{m_{1}=-j_{1}}^{j_{1}} \sum_{m_{2}=-j_{2}}^{j_{2}} \sum_{m_{3}=-j_{3}}^{j_{3}} w\left(m_{1}, m_{2}, m_{3}, u\right) \ln w\left(m_{1}, m_{2}, m_{3}, u\right)
$$

and entropies $H_{12}(u), H_{23}(u)$, and $H_{2}(u)$ are defined by means of projected tomograms

$$
\begin{array}{r}
w_{12}\left(m_{1}, m_{2}, u\right)=\sum_{m_{3}=-j_{3}}^{j_{3}} w\left(m_{1}, m_{2}, m_{3}, u\right), \\
w_{23}\left(m_{2}, m_{3}, u\right)=\sum_{m_{1}=-j_{1}}^{j_{1}} w\left(m_{1}, m_{2}, m_{3}, u\right), \\
w_{2}\left(m_{2}, u\right)=\sum_{m_{1}=-j_{1}}^{j_{1}} w_{12}\left(m_{1}, m_{2}, u\right) .
\end{array}
$$

New inequality (39) does not contradict the known strong subadditivity condition for von Neumann entropy $[18,19]$

$$
S_{123}+S_{2} \leq S_{12}+S_{23}
$$

where

$$
S_{123}=-\operatorname{Tr} \rho_{123} \ln \rho_{123},
$$

and other entropies are von Neumann entropies for reduced density matrices $\rho(1,2)=\operatorname{Tr}_{3} \rho(1,2,3)$ and $\rho(2,3)=\operatorname{Tr}_{1} \rho(1,2,3)$.

Inequalities (31) and (39) are new inequalities for composite quantum finite-dimensional systems obtained in [1]. 


\section{New inequalities for Rényi operator symbol entropies}

In this section, we continue the study of tomographic entropies along the line of our previous work [1] and derive new inequalities for spin tomographic entropies related to quantum Fourier transform. For continuous conjugate variables (position and momentum), the inequalities for Rényi entropy associated with probability densities in position and momentum were obtained in [17]. These inequalities were used to obtain new integral inequalities for symplectic and optical tomograms in [20,22-25]. In [17] for $N$-dimensional Hilbert space the analog of uncertainty relation for the Rényi entropies was given in the form

$$
\frac{1}{1-\alpha} \ln \left(\sum_{k=1}^{N} \tilde{p}_{k}^{\alpha}\right)+\frac{1}{1-\beta} \ln \left(\sum_{l=1}^{N} p_{l}^{\beta}\right) \geq \ln N
$$

where

$$
\widetilde{p}_{k}=\left|\widetilde{a}_{k}\right|^{2}, \quad p_{l}=\left|a_{l}\right|^{2}, \quad \frac{1}{\alpha}+\frac{1}{\beta}=2,
$$

and the complex numbers $\widetilde{a}_{k}$ and $a_{l}$ are connected by the quantum Fourier transform

$$
\widetilde{a}_{k}=\frac{1}{\sqrt{N}} \sum_{l=1}^{N} \exp \left(\frac{2 \pi i k l}{N}\right) a_{l} .
$$

Below we use the inequalities to obtain new inequalities for Shannon and Rényi entropies associated with unitary spin tomograms. The spin tomogram of a state with density operator $\rho$ can be considered as a column probability vector on unitary group with the components $w_{m}(u)$. Then we

can introduce another $N$-vector with components $p_{m}(u)=\sqrt{w_{m}(u)}$. Applying inequality (46)) to these vectors and the notation

$$
\left|\sum_{m^{\prime}=-j^{\prime}}^{j} F_{m m^{\prime}} \sqrt{w\left(m^{\prime}, u\right)}\right|=\sqrt{w_{F}(m, u)}
$$

where $F_{m m^{\prime}}$ is given by (9) and $w_{F}(m, u)$ is the probability distribution, we obtain inequality

$$
\frac{1}{1-\alpha} \ln \left(\sum_{m=-j}^{j} w(m, u)^{\alpha}\right)+\frac{1}{1-\beta} \ln \left(\sum_{m=-j}^{j} w_{F}(m, u)^{\beta}\right) \geq \ln N .
$$

Also using for pure state $|\psi\rangle$ the definition of spin tomogram, we obtain another similar inequality

$$
\frac{1}{1-\alpha} \ln \left(\sum_{m=-j}^{j} w(m, u)^{\alpha}\right)+\frac{1}{1-\beta} \ln \left(\sum_{m=-j}^{j} w(m, F u)^{\beta}\right) \geq \ln N
$$

where $F$ is quantum Fourier transform matrix. We can conjecture that the above inequality (51) is valued also for mixed state.

Thus one has for Rényi entropy (12) the inequality for each unitary matrix

$$
R_{\alpha}(u)+R_{\beta}(F u) \geq \ln N .
$$


Thus the unitary spin tomogram of the particle with spin $j$ for the state with $N \times N$ density matrix $\rho$, where $N=2 j+1$, must satisfy inequality (51). In the limit $\alpha \rightarrow 1, \beta \rightarrow 1$, one gets inequalities for Shannon entropy of the spin state

$$
H(u)+H(F u) \geq \ln N
$$

Another inequality reads

$$
H(u)+H_{F}(u) \geq \ln N
$$

where $H_{F}(u)$ is the Shannon entropy associated with the probability distribution $w_{F}(m, u)$.

For the minimum value of the Shannon entropy realized for unitary matrix $u_{0}$, one has the von Neumann entropy

$$
H\left(u_{0}\right)=S_{\mathrm{vN}}
$$

Inequality (53) written for $u_{0}$

$$
H\left(u_{0}\right)+H\left(F u_{0}\right) \geq \ln N
$$

provides the inequality for the von Neumann entropy

$$
S_{\mathrm{vN}}+S\left(F u_{0}\right) \geq \ln N
$$

where $S\left(F u_{0}\right)$ is a new entropy. It has the following physical meaning. If the density operator $\hat{\rho}$ of the quantum state of spin is given in the form of spectral decomposition

$$
\hat{\rho}=\sum_{q=-j}^{j} \lambda_{q}|q\rangle\langle q|
$$

one can identify the eigenstate $|q\rangle$ of the density operator $\hat{\rho}$ with "position" state. In the approach with mutually unbiased bases and Wigner function for finite Hilbert space [26,27,38-42], the states

$$
|p\rangle=\hat{F}|q\rangle,
$$

where $\hat{F}$ is the Fourier transform operator, are interpreted as "momentum" eigenstates. The matrix elements

$$
\langle p|\hat{F}| q\rangle=F_{p q}
$$

provide the matrix $F$ which coincides with the Fourier transform matrix. Thus we have the interpretation of the new inequality in the same manner as it was done in the case of continuous variables. The new entropy $S\left(F u_{0}\right)$ in (57) is the Shannon entropy for "momentum" distribution, if we identify the standard von Neumann entropy with Shannon entropy for "position" distribution.

Let us consider the example of qubit state with density matrix

$$
\rho=\left(\begin{array}{ll}
1 & 0 \\
0 & 0
\end{array}\right)
$$

Position operator $\hat{q}$ is $\sigma_{z}$ matrix and momentum operator $\hat{p}$ is $\sigma_{x}$ matrix. Two position eigenvectors $|q\rangle$ are $\left(\begin{array}{l}1 \\ 0\end{array}\right)$ and $\left(\begin{array}{l}0 \\ 1\end{array}\right)$ and two momentum eigenvectors $|p\rangle$ are $\frac{1}{\sqrt{2}}\left(\begin{array}{l}1 \\ 1\end{array}\right)$ and $\frac{1}{\sqrt{2}}\left(\begin{array}{c}1 \\ -1\end{array}\right)$. The matrix $F$ reads

$$
F=\frac{1}{\sqrt{2}}\left(\begin{array}{cc}
1 & 1 \\
1 & -1
\end{array}\right)
$$


The matrix $u_{0}=1$. Inequality (57) is saturated since

$$
S_{\mathrm{vN}}=0, \quad S(F)=\ln 2
$$

and

$$
S_{\mathrm{vN}}+S(F)=\ln 2 \geq \ln 2 .
$$

Also the inequality for Rényi entropy is saturated

$$
R_{\alpha}\left(u_{0}\right)+R_{\beta}\left(F u_{0}\right)=\ln 2 \geq \ln 2 .
$$

In the considered example, the $|q\rangle$ and $|p\rangle$ vectors form that is called "mutually unbiased bases" [39-42].

One should note that there are Shannon entropic uncertainty relations for distributions associated with set of mutually unbiased bases [15] and with pairs of orthogonal bases [12]. In the case where mutually unbiased bases are connected by quantum Fourier transform, our result (56) coincides with [12].

In [1] group average Shannon and Rényi entropies were introduced.

Due to invariance of Haar measure, one can conclude that the group average Shannon tomographic entropy satisfies the inequality

$$
\bar{H}=\int H(u) d u \geq \frac{1}{2} \ln N
$$

Also for group average Rényi entropy (12), one has

$$
\bar{R}_{\alpha \beta}=\int R_{\alpha}(u) d u+\int R_{\beta}(u) d u \geq \ln N, \quad \frac{1}{\alpha}+\frac{1}{\beta}=2 .
$$

To illustrate the inequalities obtained, let us now discuss the mixed state of qubit with diagonal density matrix

$$
\rho=\left(\begin{array}{ll}
a & 0 \\
0 & b
\end{array}\right), \quad a+b=1 .
$$

Then inequality (53) can be visualized as follows.

Von Neumann entropy of this state

$$
S_{\mathrm{vN}}=-a \ln a-b \ln b .
$$

The density matrix subjected by quantum Fourier transform (62) reads

$$
F^{\dagger} \rho F=\left(\begin{array}{cc}
1 / 2 & (a-b) / 2 \\
(a-b) / 2 & 1 / 2
\end{array}\right)
$$

Its tomographic entropy

$$
H\left(F u_{o}\right)=\ln 2, \quad u_{0}=1 .
$$

Thus inequality (53) looks as follows:

$$
-a \ln a-b \ln b+\ln 2 \geq \ln 2,
$$


which only means that von Neumann entropy is nonnegative. But inequality (54) gives better estimation since the number $\ln 2$ is replaced by a smaller number. In fact, the tomographicprobability vector of the qubit state

$$
\vec{w}=\left(\begin{array}{c}
a \\
b
\end{array}\right)
$$

is associated to the probability-amplitude vector with positive components

$$
\vec{W}=\left(\begin{array}{c}
\sqrt{a} \\
\sqrt{b}
\end{array}\right)
$$

Then after making the quantum Fourier transform of this vector, we get the column vector

$$
\vec{W}_{F}=\frac{1}{\sqrt{2}}\left(\begin{array}{c}
\sqrt{a}+\sqrt{b} \\
\sqrt{a}-\sqrt{b}
\end{array}\right) .
$$

The probability-distribution vector associated to the above probability-amplitude vector reads

$$
\vec{w}_{F}=\left(\begin{array}{c}
(1 / 2)+\sqrt{a b} \\
(1 / 2)-\sqrt{a b}
\end{array}\right) .
$$

Thus we apply inequality relating Shannon entropies to two vectors (74) and (76) and obtain

$$
-a \ln a-b \ln b-\left(\frac{1}{2}+\sqrt{a b}\right) \ln \left(\frac{1}{2}+\sqrt{a b}\right)-\left(\frac{1}{2}-\sqrt{a b}\right) \ln \left(\frac{1}{2}-\sqrt{a b}\right) \geq \ln 2,
$$

or

$$
S_{\mathrm{vN}}-\left(\frac{1}{2}+\sqrt{a b}\right) \ln \left(\frac{1}{2}+\sqrt{a b}\right)-\left(\frac{1}{2}-\sqrt{a b}\right) \ln \left(\frac{1}{2}-\sqrt{a b}\right) \geq \ln 2 .
$$

This inequality is not that obvious though we know that $S_{\mathrm{vN}} \geq 0$.

Some inequalities for unitary matrix can be obtained.

Let us consider $N \times N$-unitary matrix $u_{j k}$. One has the inequality

$$
-\sum_{j=1}^{N}\left(\left|u_{j k}\right|^{2} \ln \left|u_{j k}\right|^{2}+\left|(F u)_{j k}\right|^{2} \ln \left|(F u)_{j k}\right|^{2}\right) \geq \ln N
$$

or

$$
-\sum_{j=1}^{N} \sum_{k=1}^{N}\left(\left|u_{j k}\right|^{2} \ln \left|u_{j k}\right|^{2}+\left|(F u)_{j k}\right|^{2} \ln \left|(F u)_{j k}\right|^{2}\right) \geq N \ln N,
$$

where $F_{j k}$ is the Fourier transform matrix. Integrating inequality (79) over the unitary group with Haar measure normalized as in (3) one has the inequality

$$
-\int\left(\sum_{j=1}^{N}\left|u_{j k}\right|^{2} \ln \left|u_{j k}\right|^{2}\right) d u \geq \frac{1}{2} \ln N
$$

We demonstrated on the example of qubit that for tomograms of the spin states connected by quantum Fourier transforms one has constraints in the form of inequalities for Shannon tomographic entropies. One can demonstrate analogous constraints for Rényi tomographic entropies too. 


\section{Conclusions}

We point out that there exist several inequalities for Shannon and Rényi entropies associated to spin quantum state tomograms. These inequalities provide extra information theory constraints in addition to known subadditivity and strong subadditivity conditions. Physical and information meaning of the inequalities obtained needs extra clarification.

\section{Acknowledgments}

The study was supported by the Russian Foundation for Basic Research under Project No. 0702-00598. The authors thank the University of Lisbon where this work was initiated and partially done for hospitality. M.A.M. thanks the Organizers of the International Workshop "Nonlinear Physics. Theory and Experiment. V" (Gallipoli, Lecce, Italy, 2008) for kind hospitality and the Russian Foundation for Basic Research for Travel Grant No. 08-02-08174.

\section{Список литературы}

[1] M.A. Man'ko, V.I. Man'ko and R. Vilela Mendes, J. Russ. Laser Res. 27 (2006) 507

[2] O.V. Man'ko, V.I. Man'ko and G. Marmo, J. Phys. A: Math. Gen. 35 (2002) 699

[3] C.E. Shannon, Bell Systems Technical Journal 27 (1948) 379

[4] A. Rényi, Probability Theory (1970) North-Holland, Amsterdam

[5] W. Heisenberg, Z. Phys. 43 (1927) 172

[6] E. Schrödinger, Ber. Kgl. Akad. Wiss. Berlin 24 (1930) 296

[7] H.P. Robertson, Phys. Rev. 35 (1930) 667

[8] H.P. Robertson, Phys. Rev. bf 46 (1934) 794

[9] I.I. Hirschman, Amer. J. Math. 79 (1957) 152

[10] I. Bialynicki-Birula and J. Mycielski, Commun. Math. Phys. 44 (1975) 129

[11] V.V. Dodonov and V.I. Man'ko, Invariants and Evolution of Nonstationary Quantum Systems, Proceedings of the Lebedev Physical Institute, Nova Science, Commack, New York (1989), Vol. 183

[12] H. Maassen and J.B.M. Uffink, Phys. Rev. Lett. 60 (1988) 1103

[13] A.K. Rajagopal, Phys. Lett. A 205 (1995) 32

[14] J. Sánchez-Ruiz, Phys. Lett. A 201 (1995) 125

[15] M.A. Ballester and S. Wehner, Phys. Rev. A 75 (2007) 022319 
[16] A. Azarchs, quant-ph/0412083 v1

[17] I. Bialynicki-Birula, Phys. Rev. A 74 (2006) 052101

[18] E.H. Lieb and M.B. Ruskai, J. Math. Phys. 14 (1973)

[19] M.B. Ruskai, quant-ph/0404126 v4

[20] S. De Nicola, R. Fedele, M.A. Man'ko and V.I. Man'ko, Eur. J. Phys. B 52 (2006) 191

[21] M.A. Man'ko, J. Russ. Laser Res. 27 (2006) 405

[22] M.A. Man'ko, V.I. Man'ko, S. De Nicola and R. Fedele, Acta Phys. Hung. B 26/1-2 (2006) 71

[23] S. De Nicola, R. Fedele, M.A. Man'ko and V.I. Man'ko, Theor. Math. Phys. 152 (2007) 1081

[24] M.A. Man'ko, "Tomographic entropy and new entropic uncertainty relations," in: Quantum Theory: Reconsideration of Foundations-4 (Vaxjo, Sweden, June 2007), Editors: G. Adenier, A. Yu. Khrennikov, P. Lahti, V.I. Man'ko and T. Nieuwenhuizen, AIP Conference Proceedings Series, Vol. 962, pp. 132-139 (2007)

[25] S. De Nicola, R. Fedele, M.A. Man'ko and V.I. Man'ko,, J. Phys. Conf. Ser. 70012007 (2007)

[26] J. Schwinger, Proc. Natl. Acad. Sci. USA 46 (1960) 570 [reprinted in Quantum Kinematics and Dynamics, Benjamin, New York (1970)]

[27] S. Zhang, C. Lei, A. Vourdas, and J.A. Dunningham, J. Phys. B: At. Mol. Opt. Phys. 39 (2006) 1625

[28] P.W. Shor, quant-ph/9508027

[29] Y.S. Weinstein, M.A. Pravia, E.M. Fortunato, S. Lloyd and D.G. Cory, Phys. Rev. Lett. 86 (2001) 1889

[30] V.I. Man'ko, G. Marmo, E.C.G. Sudarshan and F. Zaccaria, Phys. Lett. A 327 (2004) 353

[31] V.V. Dodonov and V.I. Man'ko, Phys. Lett. A 239 (1997) 335

[32] V.I. Man'ko and O.V. Man'ko, J. Exp. Theor. Phys. 85 (1997) 430

[33] O.V. Man'ko and V.I. Man'ko, J. Russ. Laser Res. 25 (2004) 115

[34] D. Deutsch, Phys. Rev. Lett. 50 (1987) 631

[35] K. Kraus, Phys. Rev. D 35 (1987) 3070

[36] W.K. Wootters, Ann. Phys. 176 (1987) 1

[37] W.K. Wootters, quant-ph/0306135 v4 
[38] M.R. Kibler, Collect. Czech. Chem. Commun. 70 (2005) 771

[39] M. Planat, quant-ph/0510044 v1

[40] A.B. Klimov, C. Muñoz and J.L. Romero, J. Phys. A: Math. Gen. 39 (2006) 14471

[41] C. Cormick, E.F. Galvão, D. Gottesman, J.P. Paz and A. O. Pitteger, quant-ph/0506222 v1

[42] M. Planat and H. Rosu, Eur. Phys. J. D 36 (2005) 133 